\title{
Our experience with kite string injuries during makarsankranti festival
}

\author{
Darshil A. Vaishnav ${ }^{1}$, Rahul R Gupta ${ }^{2 *}$, Ranjankrishna. G. Aiyer ${ }^{3}$ \\ ${ }^{1} 3^{\text {rd }}$ Year Resident, ${ }^{2}$ Associate Professor, ${ }^{3}$ Professor and Head, Dept. of E.N.T and Head-Neck Surgery, Baroda Medical \\ College, S.S.G. Hospital, M.S. University, Vadodara, Gujarat, India
}

*Corresponding Author: Rahul R Gupta

Email: doctor.rahul25@gmail.com

\begin{abstract}
Kite-flying is a popular activity during the Makar Sankranti festival in Gujarat which spans over $14^{\text {th }}$ and $15^{\text {th }}$ January of every year. Kite strings are an important cause of penetrating neck trauma in two-wheeler drivers. In this study a total of 63 patients of kite string injuries over face and neck were studied between 2018 and 2020 at Shri Sayajirao General Hospital, Vadodara. These patients were divided into 3 groups according to the site of injury. Males were most commonly affected. Most patients were in the 19-40 years age group. Neck was the most frequently involved in most of the patients. Majority of the patients did not use personal protective gears. An indigenous metal wire frame used over two-wheelers also appeared to be reducing the number of injuries. We conclude that awareness can prevent these injuries to a large extent.
\end{abstract}

Keywords: Kite String, Cut Throat, Uttarayan, Makarsankranti.

Introduction

"Zomato delivery boy gets injured by Kite String"

-Headline from a local news channel in 2019

"Makar Sankranti" or "Uttarayan" is a popular festival in India especially in the central, northern and the western parts. "Uttarayan" in literal sense means, "coming towards the North". It denotes the northward migration $^{1}$ of the Sun which causes changes in the wind pattern and the eventual seasonal change. This is celebrated in Gujarat by flying kites. Kites were invented 2000 years back and were used as signals, to deliver messages and to measure distances ${ }^{2}$. Benjamin Franklin conducted experiments on electricity by flying a kite with a silk thread during a thunderstorm. Kite flying is a sport popular not just in India, Pakistan and Bangladesh but also China and Brazil. In Gujarat, flying kites during the month of January is as popular as the gully cricket. Along with popularity, it also invites a series of injuries. The sport involves flying the kite as high as possible, engaging in a duel with the opponents and cutting their strings. Right from the kite manufacturing to flying, a number of modes of injuries can be associated with it.

\section{Modes of injury}

Kite making involves making the string which is often made of cotton or nylon covered with multiple layers of ground glass called "manjha". 3 It is the "manjha" that decides the strength of the string. The person with a weak string is often ridiculed and thus people opt for stronger strings. Injuries involve those on the hands and fingers during making the strings and flying the kites, fall from heights, electric burns due to the kites getting entangled in live wires, road traffic accidents because of children running after the kites on roads and because of kite strings injuring the two-wheeler drivers ${ }^{4}$. Kite string injuries in the two wheeler drivers can prove to be fatal. At some places, e.g. Surat, flyovers are closed on the day of Uttarayan to prevent kite string injuries in two-wheeler drivers. The injury is seldom seen among pedestrians ${ }^{5,6}$. This means it is the speed that determines the extent and depth of injury. High driving speed means high force acting on a relatively small area, causing high pressure leading to penetration of the manjha into the skin, specially neck and face, by the slitting action. The role of kite strings as mode of penetrating neck injuries is seldom discussed in literature. It is this mode of injury that we have studied below.

\section{Relevant Anatomy}

Penetrating neck trauma has been classified in the literature according to the Roon and Christensen classification ${ }^{1}$ :
Fig. 1

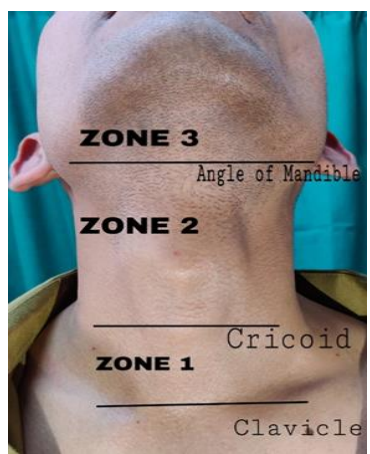

IP Journal of Otorhinolaryngology and Allied Science, January-March 2020;3(1): 1-6 
Zone 1: Extends from the clavicle to the cricoid cartilage. This region contains major vascular structures like the subclavian artery \& vein, jugular vein and common carotid artery as well as the oesophagus, trachea and thyroid.

Zone 2: Extends from the cricoid to the angle of mandible. This region contains the common carotid artery, internal and external carotid arteries, jugular vein, larynx, hypopharynx and cranial nerves X, XI \& XII.

Zone 3: Area extending from the angle of mandible to the skullbase.

\section{Methodology and management}

This study was conducted at Sri Sayajirao General Hospital, Vadodara and included patients presenting to the ENT department with kite string injuries. Patients with kite string injuries over the neck and face(excluding the eyes) were seen by the E.N.T department while the rest are seen by General Surgery department. The patients presenting to the Trauma Centre were assessed for A: Airway, B: Breathing and C: Circulation and ATLS protocols were followed. Pulse and Blood pressure were recorded. Cervical Spine involvement and a quick Neurological assessment was done to check the consciousness level of the patient. All the patients were considered to be medicolegal as the kite string can cause grievous hurt. Most of the patients had a history of road traffic accident and though all of these injuries were alleged to be accidental, an angle of homicidal and suicidal nature were also ruled out. Often the patients were referred to us from some other centre and some form of bandaging had been in place. Bandages were carefully removed keeping all preparations ready to ligate/ repair major/ minor vessel trauma, should they start bleeding. Active bleeding was stopped using clamps and pressure after adequately delineating great vessels.

A detailed history was elicited. Often the patients had been brought by ambulances from accident sites and the relatives weren't present. History of fall from vehicle, alcohol consumption, head injury, loss of consciousness, convulsions was elicited. A quick review by general surgeon as well as orthopaedic surgeon was sought to rule out injuries to other parts of the body. Many patients had kite string injuries over the fingers, arms or around ankles (as these parts were exposed and were used to disentangle the string). These were managed accordingly. Tetanus toxoid was administered. A wide bore intravenous cannula was inserted. Blood sample was taken for a quick Hemogram and Blood grouping, should the need for transfusion arise. Along with these, serum electrolytes, renal function tests, liver function tests and serological profile was also done.

\section{Triage}

Category I: Patients with injury involving only the skin, subcutaneous tissues and platysma and those with injuries to the lips, nose and ear

These patients were treated in the trauma bay. Wound was cleaned with saline and betadine, taking all aseptic precautions and after adequate haemostasis, sutured in bilayer or single layer depending on the depth. Patients with contaminated wounds were admitted, anticipating a post procedure infection while others were asked to follow-up for dressing and given oral antibiotics. Those admitted were usually discharged within 2 days. Patients with clean wounds over the nose, ear or lip region were called for regular dressing.

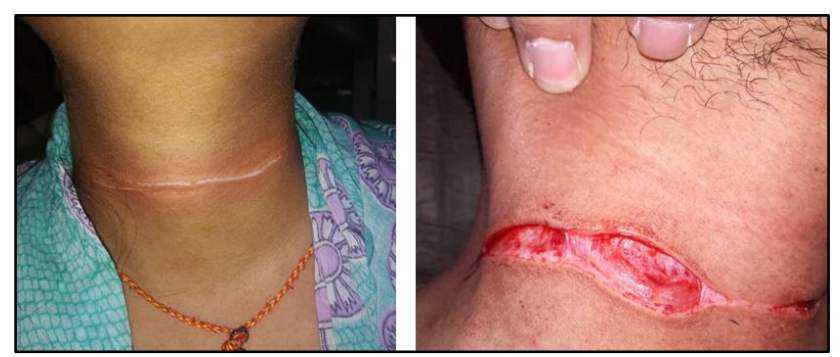

Fig. 2,3: Showing Category I injuries

\section{Category II: Patients with the injury to strap muscles and the sternocleidomastoid}

These patients were shifted to the E.N.T Operation Theater. The wound was quickly explored. If there was suspicion of a major blood vessel injury, the patient was given general anaesthesia else was managed under local anaesthesia. The wound was thoroughly cleaned. Minor bleeding vessels were cauterised. Blood clots removed. Strap muscles and sternocleidomastoid sutured using Vicryl 3-0. If Anterior Jugular vein or External Jugular vein were found to be injured, these were ligated. In such cases, a negative suction drain was also placed. Wound was closed in bilayer. These patients were usually kept indoor for 3-4 days so that development of hematoma can be watched for. The negative suction drain was removed on $3^{\text {rd }}$ or $4^{\text {th }}$ day if there was no collection. Sutures were removed on the $8^{\text {th }}$ day. 


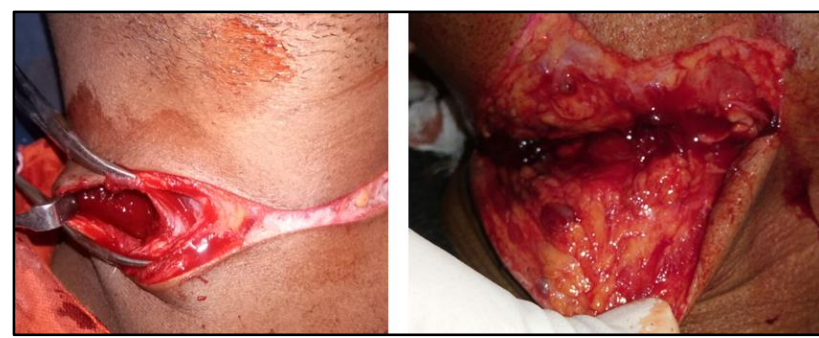

Fig.4,5: Showing Category II injuries

\section{Category III: Patients with injuries to airway}

These patients were shifted to the E.N.T Operation Theater. Hoarseness, stridor, respiratory distress, hemoptysis and subcutaneous emphysema all suggest injury to airway. Before shifting, a temporary Tracheotomy tube of appropriate size was inserted through the defect in the airway to maintain respiration and prevent aspiration. The patient was given general anaesthesia. A proper temporary tracheotomy was performed below the wound. A gap of 3 healthy Tracheal rings was planned between airway sutures and the stoma, to prevent tracheotomy infection reaching airway sutures. The defect was sutured using Prolene 2-0. Rest of the soft tissues were managed as in Category II. Flexible laryngoscopy was done to examine the lumen of the airway and to check for vocal cord mobility. The vocal cord status was documented in all cases especially as these were medicolegal cases. Patients in this category had a prolonged hospital stay. Temporary vocal cord paresis, if present, was documented. They were given Ryle's tube feeding till the laryngeal edema subsided. Decannulation was done on the $10^{\text {th }}$ day.

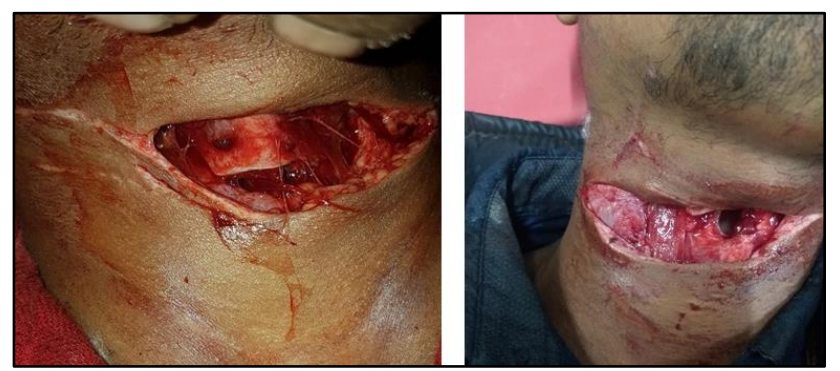

Fig. 6,7: Showing Airway injury

\section{Results and Discussion}

In this study, a total of 63 patients have been studied. All of these patients presented between January 2018 and January 2020. Among these, 54 patients were males while only 9 were females(5:1). This was reflective of the fact that more males drive twowheelers than females. This was also comparable to the study by Ventura $^{7}$ et al, in which Male: Female ratio was $12: 1.47(75 \%)$ patients presented on the $14^{\text {th }}$ and the $15^{\text {th }}$ of January, which are the days that see maximum number of kites in the sky. This was comparable to the study by Gupta et al, in which maximum patients had presented on these two days. ${ }^{1}$ $8(13 \%)$ patients presented in the week preceding Uttarayan while $4(6 \%)$ presented on the weekend after Uttarayan, suggesting the craze of kite flying among youngsters starts early before the actual festival and lasts till about 1 week after the actual celebrations are over. $3(5 \%)$ patients were very unlucky, because they suffered injuries in the months of November and December, when the number of kites flying is scarce.

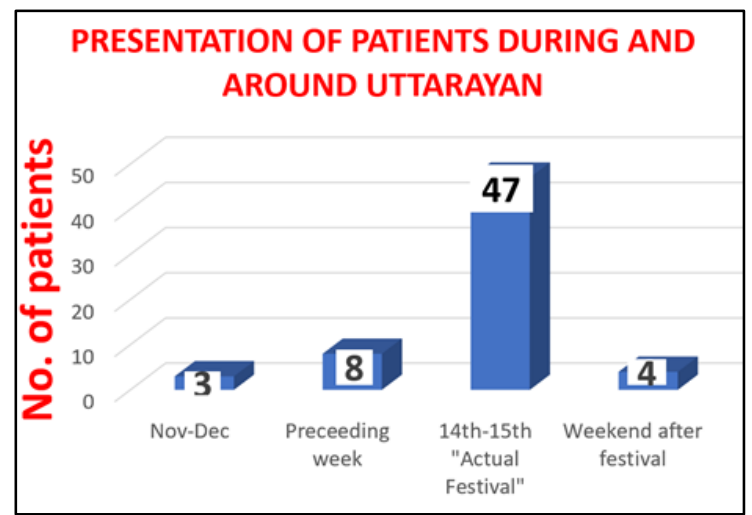

Graph. 1: Presentation of patients during and around uttarayan

As seen below, $78 \%$ of the patients were between the ages 19 and 40 years. ${ }^{8}$ This was comparable with the study by Gupta et al in which most of the patients were in 16-25 years age group. The youngest patient was 4 years while the oldest patient was 65 years old.

\begin{tabular}{|c|c|c|c|c|}
\hline Age(Years) & $<\mathbf{1 8}$ & $\mathbf{1 9 - 4 0}$ & $\mathbf{4 1 - 6 0}$ & $\mathbf{> 6 0}$ \\
\hline No. of Patients & 8 & 49 & 5 & 1 \\
\hline
\end{tabular}

Fortunately, none of the patients in the paediatric age group had airway injury or great blood vessel injury. All our patients were riding on a fast moving two wheeler, often a motorcycle and even a bicycle in one case. 50(79\%) patients were driving the vehicles while others were riding pillion. It is worth mentioning that 3 children were sitting in front of the two-wheeler driver and thus got caught up in the kite strings. It was also observed that injuries to the ear were more common in pillion riders. Only 2(3\%) out of 63 patients were wearing a helmet while only $1(1.6 \%)$ patient had the guarding wire frame installed in front of their vehicles. Considering that on an average the wire frame usage reached upto $50 \%$ of all two-wheelers, there was a marked and statistically significant reduction in injuries in riders using this frame. $2(3 \%)$ patients were 
wearing scarves over the neck, despite which the injury occurred, demonstrating the penetrating nature of the kite string. One patient wearing the helmet was unlucky to have sustained injury over the nose as the kite string got stuck in the gap in the helmet as he did not have a visor screen, while the other one got injured over the unprotected neck.

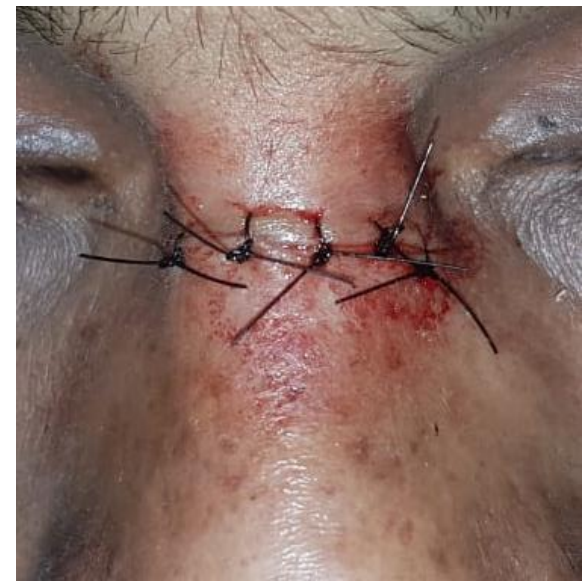

Fig. 8: showing injury over nose despite using helmet.

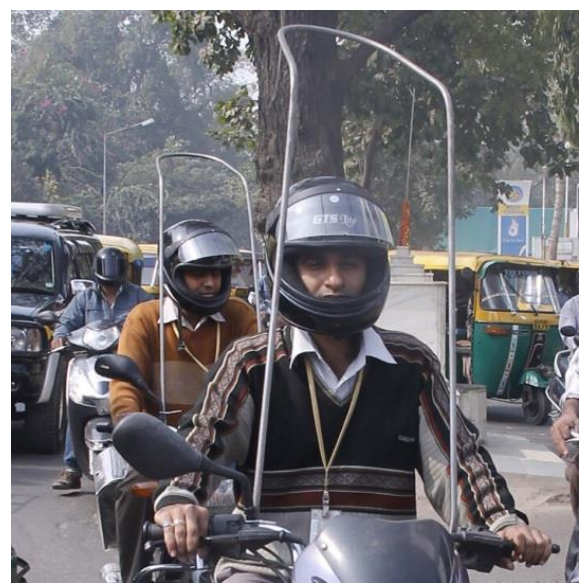

Fig. 9: Showing protective frame mounted in front of the vehicle

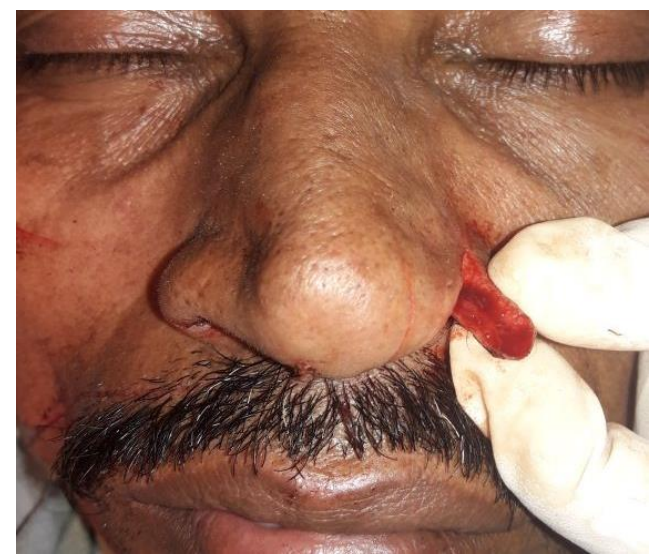

Fig. 10: Showing injury over nasal ala

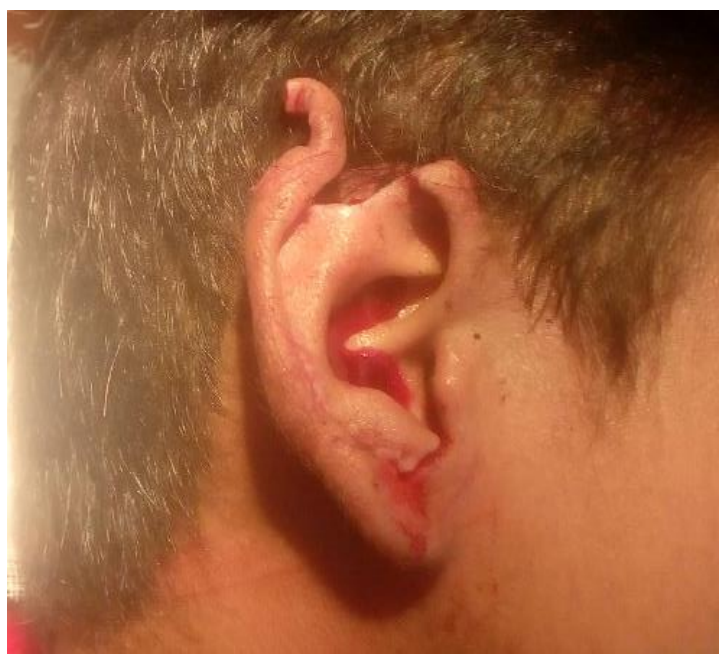

Fig. 11: Showing injury over pinna
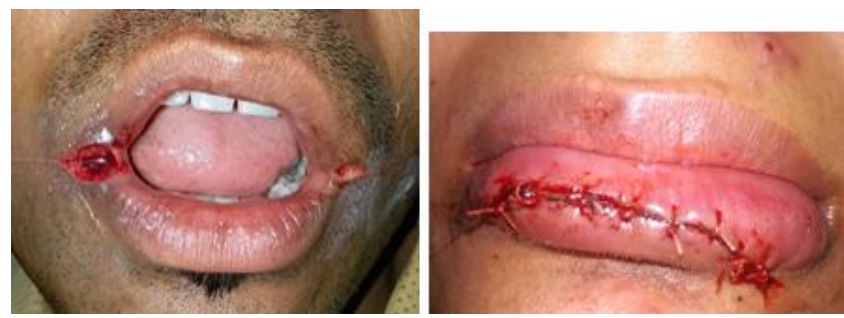

Fig. 12,13: Showing injury to angle of mouth and lips

\begin{tabular}{|l|c|}
\hline \multicolumn{1}{|c|}{ Depth of neck injury } & $\begin{array}{c}\text { Number of } \\
\text { patients }\end{array}$ \\
\hline Skin & 3 \\
\hline Skin and Subcutaneous tissue and Platysma & 28 \\
\hline Strap Muscles & 24 \\
\hline SCM & 19 \\
\hline Vessels & 10 \\
\hline Airway & 6 \\
\hline
\end{tabular}

\begin{tabular}{|l|c|}
\hline & Number of patients \\
\hline Neck & 50 \\
\hline Ear & 04 \\
\hline Nose & 04 \\
\hline Lips and cheeks & 05 \\
\hline Total & $\mathbf{6 3}$ \\
\hline
\end{tabular}

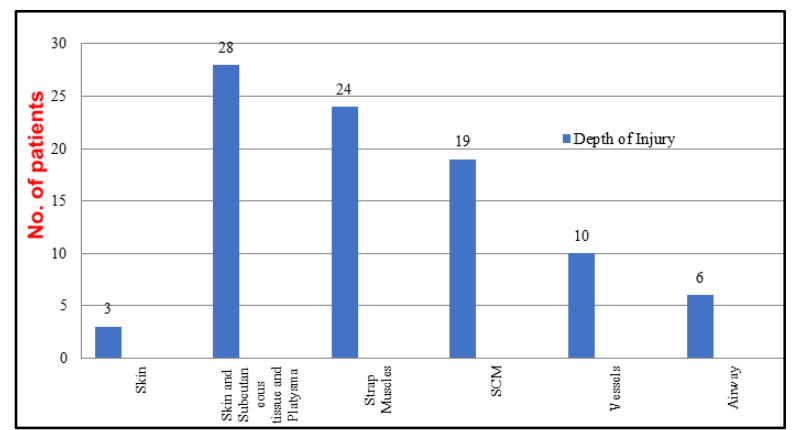

Graph 2: Depth of injury neck injury v/s No. of Patients 
As seen above, most patients had injury to the Skin, subcutaneous tissues, platysma, strap muscles and the Sternocleidomastoid. Only Skin was involved in $3(5 \%)$ patients while only skin, subcutaneous tissue upto platysma were involved in $28(44 \%)$ patients. This was comparable to the study by Gupta et al, in which injury to these structures was found to be the most common $^{1}$. In 8 patients, Anterior Jugular vein was found to be injured while in 2 patients, the External Jugular vein was injured. The Internal Jugular Vein, the Common Carotid, the Internal and External Carotid arteries were not damaged in any of the patients. $6(9.5 \%)$ patients had injury to the airway. On detailed examination, 3 patients had a tear involving the Cricothyroid membrane, 2 patients had a tear in the Cricotracheal membrane while 1 patient had injury to the $1^{\text {st }}$ tracheal ring. In all these patients, a temporary tracheotomy was done. One of these was a delivery boy from a popular food delivery service which got huge media attention and brought the problem into good limelight. 2 patients presented to us with a sutured wound but with swelling under the suture line. Clinical examination and ultrasonography revealed collection underneath. On removal of the sutures, hematoma was found. There was active oozing from small blood vessels. These were ligated and the wound was sutured. None $(0 \%)$ of the patients with injury to vessels or airway used the protective wire frame or helmets or scarves.

Pharynx and Oesophagus were intact in all the patients. None of the patients had serious head injuries.

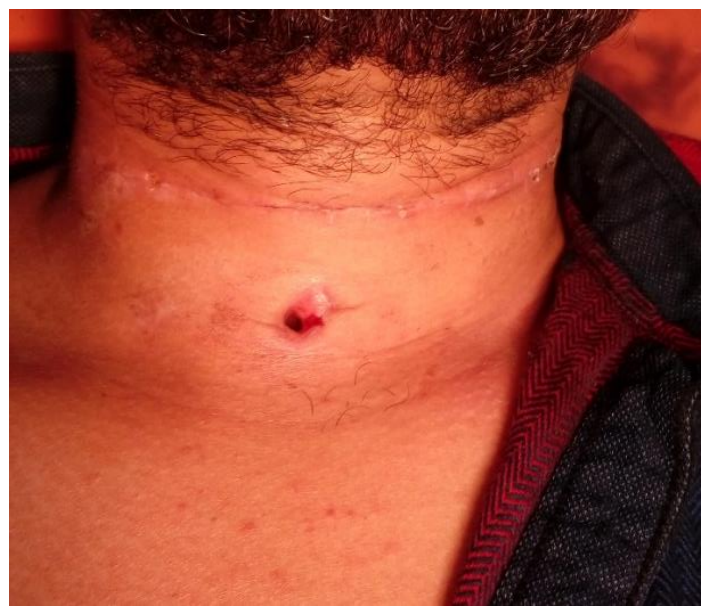

Fig. 14: Showing healed neck wound after decannulation.

\section{Sequelae}

1. Hematoma was seen in 3 patients after the initial wound suturing.
2. Temporary Tracheostomy was required in 6 patients with the airway injury to overcome extensive laryngeal edema.

3. A temporary Vocal cord palsy was seen in 3 patients. It got resolved with 15-20 days after giving steroids.

4. One patient presented to us in February 2018 with a history of kite string injury before 1 month with history of tracheostomy and subsequent strapping. He had developed suprastomal granulations, which were removed.

5. There was no mortality in our patients.

\section{Conclusions}

While kite flying is a popular activity, it can turn into a nightmare for many people like two wheeler drivers. The usage of protective devices like helmets, mufflers and jackets can prevent such injuries. An indigenously built wire frame which has become quite popular during the last couple of years, seems to be having a protective effect. In Gujarat many NGOs provide free fixation of the wire frame. Awareness programs lead by NGOs and government can be beneficial. Kite flying should be allowed only in open areas like community grounds, beaches. Riding vehicles in the crowded old city and over flyovers should be prohibited on the day of Uttarayan. Improvement in primary and secondary health care system can lead to faster management of such injuries.

\section{Source of funding}

None.

\section{Conflict of interest}

None.

\section{References}

1. Gupta RR, Aiyer RG, Gajjar Y, Jagtap P, Raval J. “Our Experience Of Kite String Injuries During "Makar Sankranti" Festival”, Gujarat Med J 2014;69:67-9.

2. Roy, Dev \& Roy, Biswanath \& Bose, Arijit. Life threatening cut throat injury due to kite string. Bangladesh Med J Khulna 20107;49;31. 10.3329/bmjk.v49i1-2.31823.

3. Babu, Annu, Harshit Garg, Sushma Sagar, Piyush Ranjan and Maneesh Singhal. "Manja Injury: A Dangerous Mechanism Cervical Injury 2015.

4. Mir MA, Ali AM, Yaseen M, Khan AH. Hand Injuries by the Killer Kite Manja and Their Management. World $J$ Plast Surg 2017;6(2):225-9.

5. Singh V, Puri P, Agrawal A, Kumar P, Singhal R. Kite String: An unsual mode of maxillofacial injury. J Indian Soc Pedod Prev Dent 2013;31:188-90.

6. Muvalia G, Jamshed N, Sinha T P, Bhoi S. Kite-string injuries: A case series. Inj J Crit Illn Inj Sci 2019;9(3):14750 . 
7. Ventura J, "Glass-coated kites and cervical injuries: a serious threat to children and adults" 923-7.

8. Gupta P \& Jain A, Patil A, Thakor R, Kumar S. Kite string injury: a thin line between harmless sport and grievous injury. Int J Comm Med Public Health 2018;10.18203/2394-6040.ijcmph20182418.
How to cite: Vaishnav DA, Gupta RR, Aiyer RG. Our experience with kite string injuries during makarsankranti festival. IP J Otorhinolaryngol Allied Sci 2019;3(1):1-6. 